\title{
Summary statistics for inhomogeneous marked point processes
}

\author{
O. Cronie - M. N. M. van Lieshout
}

Received: 15 July 2014 / Revised: 27 January 2015 / Published online: 14 March 2015

(C) The Institute of Statistical Mathematics, Tokyo 2015

\begin{abstract}
We propose new summary statistics for intensity-reweighted moment stationary marked point processes with particular emphasis on discrete marks. The new statistics are based on the $n$-point correlation functions and reduce to cross $J$ - and $D$-functions when stationarity holds. We explore the relationships between the various functions and discuss their explicit forms under specific model assumptions. We derive ratio-unbiased minus sampling estimators for our statistics and illustrate their use on a data set of wildfires.
\end{abstract}

Keywords Generating functional - Intensity-reweighted moment stationarity • $J$-function - Marked point process - Multivariate point process $\cdot$ Nearest neighbour distance distribution function $\cdot n$-point correlation function $\cdot$ Reduced Palm measure

\section{Introduction}

The analysis of a marked point pattern typically begins with computing some summary statistics which may be used to find specific structures in the data and suggest suitable models (Chiu et al. 2013; Daley and Vere-Jones 2003, 2008; Gelfand et al. 2010; Illian

This research was supported by the Netherlands Organisation for Scientific Research NWO (613.000.809) and carried out while Cronie was at CWI.

O. Cronie

Department of Mathematics and Mathematical Statistics, Umeå university, SE-901 87 Umeå, Sweden e-mail: Ottmar.Cronie@math.umu.se

M. N. M. van Lieshout $(\varangle)$

CWI, P.O. Box 94079, NL-1090 GB Amsterdam, The Netherlands

e-mail: Marie-Colette.van.Lieshout@cwi.nl; M.N.M.van.Lieshout@cwi.nl

M. N. M. van Lieshout

Department of Applied Mathematics, University of Twente, P.O. Box 217, 7500 AE Enschede,

The Netherlands 
et al. 2008; van Lieshout 2000). The choice of summary characteristic depends both on the pattern at hand and on the feature or hypothesis of interest. Indeed, under the working assumption of stationarity, for discrete marks, cross versions of the $K$ - or nearest neighbour distance distribution function may be appropriate (Diggle 2014); for real-valued marks, the mark correlation functions of Penttinen and Stoyan (1989) are widely used. Various types of $J$-functions (van Lieshout 2006; van Lieshout and Baddeley 1999) offer useful alternatives.

Often, however, the assumption of homogeneity cannot be justified. In the unmarked case, Baddeley et al. (2000) proposed an inhomogeneous extension of the $K$-function for so-called second-order intensity-reweighted stationary point processes. Their ideas were extended to spatio-temporal point processes in Gabriel and Diggle (2009), Møller and Ghorbani (2012), whereas Cronie and van Lieshout (2015), van Lieshout (2011) extended the $J$-function under the somewhat stronger assumption of intensityreweighted moment stationarity in space and time.

For non-stationary multivariate point processes, Møller and Waagepetersen (2004) proposed an extension of the $K$-function under the assumption of second-order intensity-reweighted stationarity. As we will indicate in this paper, this structure may be extended to $K$-functions for general marked point processes.

Regarding $J$-functions, in van Lieshout (2011), the author noted that the ideas in that paper could be combined with those in van Lieshout (2006) to define inhomogeneous $J$-functions with respect to mark sets. In this paper we do so, and, as a by-product, obtain a generalisation of the cross nearest neighbour distance distribution function.

The paper is structured as follows. In Sect. 2, we define marked point processes with locations in Euclidean spaces and give the necessary preliminaries. In Sects. 3.1 and 3.2 , we define, respectively, cross $D$ - and $J$-functions for inhomogeneous multivariate point processes and propose generalisations to point processes with real-valued marks. We show that $D$ and $J$ can be expressed in terms of the generating functional and discuss the relationships between these statistics and the cross $K$-function. In Sect. 4 , we investigate the form of our statistics under various independence and marking assumptions. We derive minus sampling estimators and discuss tests in Sect. 5, which are applied to a data set on wildfires in New Brunswick, Canada, in Sect. 6. We finish the paper with a summary.

\section{Definitions and notations}

Throughout this paper, we consider marked point processes $Y$ in the sense of Definition 6.4.1 in Daley and Vere-Jones (2003), with points in $\mathbb{R}^{d}$ equipped with the Euclidean metric and Borel $\sigma$-algebra $\mathcal{B}\left(\mathbb{R}^{d}\right)$. We write $\ell$ for the Lebesgue measure on $\mathcal{B}\left(\mathbb{R}^{d}\right)$. By definition, the ground process $Z$ obtained from $Y$ by ignoring the marks is a well-defined point process on $\mathbb{R}^{d}$ in its own right. We shall assume that $Z$ is simple, that is, almost surely does not contain multiple points.

We assume that the mark space $\mathcal{M}$ is Polish and equipped with a finite reference measure $v$ on the Borel $\sigma$-algebra $\mathcal{B}(\mathcal{M})$. We denote by $\mathcal{B}\left(\mathbb{R}^{d} \times \mathcal{M}\right)$ the Borel $\sigma$ algebra on the product space $\mathbb{R}^{d} \times \mathcal{M}$. In the special case that $\mathcal{M}$ is finite, $Y$ can be seen as a multivariate point process $\left(Y_{1}, \ldots, Y_{k}\right)$, where $Y_{i}$ contains the points marked $i \in \mathcal{M}=\{1, \ldots, k\}$. 


\subsection{Product densities}

Recall that the intensity measure of a marked point process is defined on product sets $A=B \times C \in \mathcal{B}\left(\mathbb{R}^{d} \times \mathcal{M}\right)$ by

$$
\Lambda(A)=\mathbb{E} Y(A)=\mathbb{E} Y(B \times C),
$$

the expected number of points in $B$ with marks in $C$. If $\Lambda$ is locally finite as a setfunction, it can be extended to a measure on $\mathcal{B}\left(\mathbb{R}^{d} \times \mathcal{M}\right)$ (see e.g. Theorem A, p. 54, in Halmos (1974)). In this paper, additionally, we assume that $\Lambda$ admits a density $\lambda$ with respect to $\ell \times v$, which is referred to as the intensity function. In particular, for a finite mark space, $\lambda(z, i) v(i)=\lambda_{i}(z)$ is the intensity function of $Y_{i}$.

Since for fixed $C$, the measure $\Lambda(\cdot \times C)$ is absolutely continuous with respect to the intensity measure $\Lambda_{g}$ of the ground process,

$$
\Lambda(B \times C)=\int_{B} M^{z}(C) \Lambda_{g}(\mathrm{~d} z) .
$$

Here $M^{z}(C)$ is the probability that the mark of a point at location $z$ falls in $C$. The members of the family $\left\{M^{z}: z \in \mathbb{R}^{d}\right\}$ of probability distributions on the Borel sets of $\mathcal{M}$ are called mark distributions.

If $Y$ is stationary, that is, if its distribution is invariant under translations of the locations, $\Lambda(B \times C)=\lambda v_{M}(C) \ell(B)$ for some probability distribution $v_{M}$ on $\mathcal{M}$, which is known as the mark distribution. In this case, we may take $v=v_{M}$ for the reference measure on $\mathcal{M}$ so that $\Lambda$ has constant intensity function $\lambda$ with respect to $\ell \times v_{M}$, and, moreover, $\lambda$ is the intensity of the ground process.

Higher order 'intensity functions' or product densities can be defined as densities $\rho^{(n)}$ of the factorial moment measures provided these exist, in which case they satisfy the following $n$th order Campbell formula. For any measurable function $f \geq 0$, the sum of $f$ over $n$-tuples of different points of $Y$ is a random variable with expectation

$$
\begin{aligned}
& \mathbb{E}\left[\sum_{\left(z_{1}, m_{1}\right), \ldots,\left(z_{n}, m_{n}\right) \in Y}^{\neq} f\left(\left(z_{1}, m_{1}\right), \ldots,\left(z_{n}, m_{n}\right)\right)\right] \\
& =\int \cdots \int f\left(\left(z_{1}, m_{1}\right), \ldots,\left(z_{n}, m_{n}\right)\right) \rho^{(n)}\left(\left(z_{1}, m_{1}\right), \ldots,\left(z_{n}, m_{n}\right)\right) \prod_{i=1}^{n} \mathrm{~d} z_{i} \mathrm{~d} v\left(m_{i}\right)
\end{aligned}
$$

(with the left hand side being infinite if and only if the right hand side is infinite). Note that $\rho^{(1)}=\lambda$, the intensity function. Also, $n$-point mark distributions $M^{z_{1}, \ldots, z_{n}}\left(C_{1} \times\right.$ $\cdots \times C_{n}$ ) can be defined analogously to the case $n=1$. For further details, see for example the textbook Chiu et al. (2013). Note that, by the absolute continuity underlying the existence of $\rho^{(n)}$, there exist product densities $\rho_{g}^{(n)}\left(z_{1}, \ldots, z_{n}\right)$ for the ground process and densities $f_{z_{1}, \ldots, z_{n}}$ of $M^{z_{1}, \ldots, z_{n}}$ with respect to the $n$-fold product of $v$ with itself such that 


$$
M^{z_{1}, \ldots, z_{n}}\left(C_{1} \times \cdots \times C_{n}\right)=\int_{C_{1}} \cdots \int_{C_{n}} f_{z_{1}, \ldots, z_{n}}\left(m_{1}, \ldots, m_{n}\right) \prod_{i=1}^{n} \mathrm{~d} v\left(m_{i}\right) .
$$

In particular, the intensity function of the ground process is given by $\lambda_{g}(z)=\rho_{g}^{(1)}(z)$ and $\lambda(z, m)=f_{z}(m) \lambda_{g}(z)$.

We will also need the related concept of n-point correlation functions $\xi_{n}, n \geq 1$, the intensity-reweighted densities of the factorial cumulant measures discussed in Section 9.5 of Daley and Vere-Jones (2008). These permutation invariant measurable functions are defined by the following recursive relation (see e.g. van Lieshout (2006), White (1979)). Set $\xi_{1} \equiv 1$ and, for $n \geq 2$,

$$
\sum_{k=1}^{n} \sum_{E_{1}, \ldots, E_{k}} \prod_{j=1}^{k} \xi_{\left|E_{j}\right|}\left(\left\{\left(z_{i}, m_{i}\right): i \in E_{j}\right\}\right)=\frac{\rho^{(n)}\left(\left(z_{1}, m_{1}\right), \ldots,\left(z_{n}, m_{n}\right)\right)}{\lambda\left(z_{1}, m_{1}\right) \cdots \lambda\left(z_{n}, m_{n}\right)},
$$

where $\sum_{E_{1}, \ldots, E_{k}}$ is a sum over all possible $k$-sized partitions $\left\{E_{1}, \ldots, E_{k}\right\}, E_{j} \neq \emptyset$, of the set $\{1, \ldots, n\}$ and $\left|E_{j}\right|$ denotes the cardinality of $E_{j}$. Note that for a Poisson process, $\xi_{n} \equiv 0$ for all $n \geq 2$.

\subsection{Palm measures}

Let $Y$ be a simple marked point process whose intensity function exists. The summary statistics in this paper are defined in terms of reduced Palm measures satisfying the reduced Campbell-Mecke formula which states that, for any measurable function $f \geq 0$,

$$
\mathbb{E}\left[\sum_{(z, m) \in Y} f((z, m), Y \backslash\{(z, m)\})\right]=\int_{\mathbb{R}^{d}} \int_{\mathcal{M}} \mathbb{E}^{!(z, m)}[f((z, m), Y)] \lambda(z, m) \mathrm{d} z \mathrm{~d} v(m)
$$

(with the left hand side being infinite if and only if the right hand side is infinite). The probability measure $P^{!(z, m)}$ corresponding to $\mathbb{E}^{!(z, m)}$ can be interpreted as the conditional probability of $Y \backslash\{(z, m)\}$ given that $Y(\{(z, m)\})=1$. For further details, see Daley and Vere-Jones (2008).

A few remarks are in order. First, consider the special case that $Y$ is stationary and the reference measure on $\mathcal{M}$ is the mark distribution $v_{M}$. In this case, it is possible to define reduced Palm measures with respect to arbitrary mark sets. Specifically, for $C \in \mathcal{B}(\mathcal{M})$ such that $v(C)=v_{M}(C)>0$, set

$$
P_{C}^{! z}(R)=\frac{1}{v(C)} \int_{C} P^{!(z, m)}(R) \mathrm{d} v(m) .
$$

Then, $P_{C}^{! z}$ does not depend on the choice of $z \in \mathbb{R}^{d}$ and is a probability measure (Section 4.4.8 in Chiu et al. (2013)). It can be interpreted as the conditional distribution of $Y$ on the complement of $\{z\} \times \mathcal{M}$, given that $Y$ places a point at $z$ with mark in $C$. 
As a second example, consider multivariate point processes $\left(Y_{1}, \ldots, Y_{k}\right)$ and let $v$ be any finite measure on $\mathcal{M}=\{1, \ldots, k\}$. Now, we have a family of reduced Palm measures $P^{!(z, i)}$ for $i=1, \ldots, k$ and may restrict ourselves to sets of the form $C=\{i\}$. Then, (5) reads

$$
P_{C}^{! z}(R)=\frac{1}{v(i)} v(i) P^{!(z, i)}(R)=P^{!(z, i)}(R)
$$

and does not depend on the specific choice of $v$.

For non-finite mark spaces, the reference measure $v$ on $\mathcal{M}$ may not correspond to a well-defined mark distribution. One pragmatic approach is to take a finite partition of the mark space, $\mathcal{M}=\cup_{i=1}^{k} \mathcal{M}_{i}$, and proceed as in the multivariate case. An alternative is to use (5) as definition for a $v$-averaged reduced Palm distribution with respect to $C$, bearing in mind that the definition does depend on the choice of $v$.

\subsection{Generating functionals}

When product densities of all orders exist, the generating functional $G(\cdot)$, which uniquely determines the distribution of $Y$ (see e.g. Theorem 9.4.V, Daley and Vere-Jones 2008), is defined as follows. For all mappings $v=1-u$ such that $u: \mathbb{R}^{d} \times \mathcal{M} \rightarrow[0,1]$ is measurable with bounded support, set

$$
\begin{aligned}
G(v)= & G(1-u)=\mathbb{E}\left[\prod_{(z, m) \in Y} v(z, m)\right] \\
= & 1+\sum_{n=1}^{\infty} \frac{(-1)^{n}}{n !} \int_{\mathbb{R}^{d} \times \mathcal{M}} \cdots \int_{\mathbb{R}^{d} \times \mathcal{M}} \rho^{(n)}\left(\left(z_{1}, m_{1}\right), \ldots,\left(z_{n}, m_{n}\right)\right) \\
& \times \prod_{i=1}^{n} u\left(z_{i}, m_{i}\right) \mathrm{d} z_{i} \mathrm{~d} v\left(m_{i}\right) \\
= & \exp \left[\sum_{n=1}^{\infty} \frac{(-1)^{n}}{n !} \int_{\mathbb{R}^{d} \times \mathcal{M}} \cdots \int_{\mathbb{R}^{d} \times \mathcal{M}} \xi_{n}\left(\left(z_{1}, m_{1}\right), \ldots,\left(z_{n}, m_{n}\right)\right)\right. \\
& \left.\times \prod_{i=1}^{n} u\left(z_{i}, m_{i}\right) \lambda\left(z_{i}, m_{i}\right) \mathrm{d} z_{i} \mathrm{~d} v\left(m_{i}\right)\right] .
\end{aligned}
$$

By convention, $\log 0=-\infty$ and an empty product equals 1 . The last equalities hold provided that the right hand sides converge (see e.g. p. 126, Chiu et al. 2013). Similarly, for $a \in \mathbb{R}^{d}$ and $C \in \mathcal{B}(\mathcal{M})$, we may define the generating functional $G_{C}^{! a}$ with respect to $P_{C}^{! a}$ (see the discussion around (5)) by

$$
G_{C}^{! a}(v)=\frac{1}{v(C)} \int_{C} \mathbb{E}^{!(a, b)}\left[\prod_{(z, m) \in Y} v(z, m)\right] \mathrm{d} v(b) .
$$




\section{Definition of summary statistics}

\subsection{Inhomogeneous cross $D$-function}

In this section, we define cross $D$-functions for marked point processes in analogy with the inhomogeneous nearest neighbour distance distribution function of van Lieshout (2011).

Write

$$
\bar{\lambda}_{E}=\inf _{z \in \mathbb{R}^{d}, m \in E} \lambda(z, m) .
$$

Throughout we assume that $Y$ is a simple marked point process whose product densities of all orders exist and for which the $\xi_{n}, n \geq 2$, are translation invariant in the sense that

$$
\xi_{n}\left(\left(z_{1}+a, m_{1}\right), \ldots,\left(z_{n}+a, m_{n}\right)\right)=\xi_{n}\left(\left(z_{1}, m_{1}\right), \ldots,\left(z_{n}, m_{n}\right)\right)
$$

for all $a \in \mathbb{R}^{d}$ and $\ell \otimes v$-almost all $\left(z_{i}, m_{i}\right) \in \mathbb{R}^{d} \times \mathcal{M}$. If, moreover, $\bar{\lambda}=\bar{\lambda}_{\mathcal{M}}>0$, then $Y$ is said to be intensity-reweighted moment stationary (IRMS). This definition is due to van Lieshout (2011). In the context of multivariate point processes, a variation in which only $\xi_{2}$ is required to be translation invariant can be found in Møller and Waagepetersen (2004).

Clearly, if $Y$ is stationary, it is also IRMS. Assuming that the intensities are bounded away from zero, further examples include Poisson processes, multivariate point processes with independent IRMS components (cf. Proposition 4.4 in Møller and Waagepetersen (2004)) or intensity-reweighted moment stationary point processes that are independently marked (cf. (16)). Since the $\xi_{n}$ are invariant under independent thinning, further examples include such location or mark-dependent thinnings of stationary point processes. The special case of spatio-temporal point processes is treated in detail in Cronie and van Lieshout (2015).

Definition 1 Let $Y$ be IRMS and let $C$ and $E$ be Borel sets in $\mathcal{M}$ with $v(C)$ and $v(E)$ strictly positive. Write $B(a, r)$ for the closed ball centred at $a$ with radius $r$. Set

$$
u_{r}^{a, E}(z, m)=\frac{\bar{\lambda}_{E} \mathbf{1}\{(z, m) \in B(a, r) \times E\}}{\lambda(z, m)}, \quad a \in \mathbb{R}^{d}, \quad E \in \mathcal{B}(\mathcal{M}),
$$

and define, for $r \geq 0$, the inhomogeneous cross nearest neighbour distance distribution function by

$$
\begin{aligned}
& D_{\text {inhom }}^{C E}(r)=1-G_{C}^{! 0}\left(1-u_{r}^{0, E}\right) \\
& \quad=1-\frac{1}{v(C)} \int_{C} \mathbb{E}^{!(0, b)}\left[\prod_{(z, m) \in Y}\left(1-\frac{\bar{\lambda}_{E} \mathbf{1}\{(z, m) \in B(0, r) \times E\}}{\lambda(z, m)}\right)\right] \mathrm{d} v(b) .
\end{aligned}
$$


We shall show in Theorem 1 below that the specific choice $a=0$ in (8) is merely a matter of convenience. Moreover, $\bar{\lambda}_{E}$ may be replaced by smaller strictly positive scalars.

When $Y$ is stationary and $v=v_{M}$, the mark distribution,

$$
\begin{aligned}
D_{\text {inhom }}^{C E}(r) & =1-\frac{1}{v_{M}(C)} \int_{C} \mathbb{E}^{!(0, b)}\left[\prod_{(z, m) \in Y} \mathbf{1}\{(z, m) \notin B(0, r) \times E\}\right] \mathrm{d} v_{M}(b) \\
& =P_{C}^{! 0}(Y \cap B(0, r) \times E \neq \emptyset),
\end{aligned}
$$

so that (8) reduces to the $C$-to- $E$ nearest neighbour distance distribution function of van Lieshout (2006) for marked point processes.

\subsubsection{Multivariate point process}

Consider a multivariate point process $Y=\left(Y_{1}, \ldots, Y_{k}\right)$ that is intensity-reweighted moment stationary. Let $C=\{i\}$ and $E=\{j\}$ for $i \neq j \in\{1, \ldots, k\}$. Write $\bar{\lambda}_{j}=$ $\inf _{z \in \mathbb{R}^{d}} \lambda_{j}(z)$ and note that $\bar{\lambda}_{E} / \lambda(z, j)$ is equal to $\bar{\lambda}_{j} / \lambda_{j}(z)$. Therefore, (8) reduces to

$$
D_{\text {inhom }}^{i j}(r)=1-\mathbb{E}^{!(0, i)}\left[\prod_{z \in Y_{j}}\left(1-\frac{\bar{\lambda}_{j}}{\lambda_{j}(z)} \mathbf{1}\{z \in B(0, r)\}\right)\right]
$$

which under the further assumption that $Y$ is stationary is equal to

$$
P^{!(0, i)}\left(Y_{j} \cap B(0, r) \neq \emptyset\right),
$$

the classical cross nearest neighbour distance distribution function, see e.g. Chapter 21 in Gelfand et al. (2010). If $Y$ is a Poisson process,

$$
D_{\text {inhom }}^{i j}(r)=1-\exp \left[-\bar{\lambda}_{j} \ell(B(0, r))\right]
$$

Smaller values of $D_{\text {inhom }}^{i j}(r)$ suggest that there are fewer points of type $j$ in the $r$ neighbourhood, that is, inhibition; larger values indicate that points of type $j$ are attracted by those of type $i$ at range $r$. In the case $i=j$, we obtain the inhomogeneous $D$-function of $Y_{i}$.

With $C=\{i\}$ for some $i \in\{1, \ldots, k\}$ and $E=\mathcal{M}=\{1, \ldots, k\},(8)$ is equal to

$$
D_{\text {inhom }}^{i \bullet}(r)=1-\mathbb{E}^{!(0, i)}\left[\prod_{(z, m) \in Y}\left(1-\frac{\bar{\lambda} \mathbf{1}\{z \in B(0, r)\}}{\lambda(z, m)}\right)\right]
$$

for $r \geq 0$. Note that the function $u_{r}^{0, \mathcal{M}}$ may depend on $v$ through $\lambda(z, m)$. If we give equal weight to each member of $\mathcal{M}$, however, $\bar{\lambda} / \lambda(z, m)=\tilde{\lambda} / \lambda_{m}(z)$ is uniquely 
defined in terms of the intensity functions of the components of $Y$ and the minimal marginal intensity $\tilde{\lambda}=\inf \left\{\lambda_{i}(z): z \in \mathbb{R}^{d}, i \in\{1, \ldots, k\}\right\}$. If $Y$ is stationary, $D_{\text {inhom }}^{i \bullet}$ is the classic $i$-to-any nearest neighbour distance distribution function.

\subsection{Inhomogeneous cross $J$-functions}

In this section, we define cross $J$-functions for marked point processes in analogy with the inhomogeneous $J$-function of van Lieshout (2011). Throughout we assume that $Y$ is a simple intensity-reweighted moment stationary point process.

Definition 2 Let $Y$ be IRMS and let $C$ and $E$ be Borel sets in $\mathcal{M}$ with $v(C)$ and $v(E)$ strictly positive. For $r \geq 0$ and $n \geq 1$, set

$$
\begin{aligned}
J_{n}^{C E}(r)= & \int_{C} \int \cdots \int_{(B(0, r) \times E)^{n}} \xi_{n+1}\left((a, b),\left(z_{1}+a, m_{1}\right), \ldots,\left(z_{n}+a, m_{n}\right)\right) \\
& \times \mathrm{d} v(b) \prod_{i=1}^{n} \mathrm{~d} z_{i} \mathrm{~d} v\left(m_{i}\right)
\end{aligned}
$$

and define the inhomogeneous cross $J$-function by

$$
J_{\text {inhom }}^{C E}(r)=\frac{1}{v(C)}\left(v(C)+\sum_{n=1}^{\infty} \frac{\left(-\bar{\lambda}_{E}\right)^{n}}{n !} J_{n}^{C E}(r)\right)
$$

for all ranges $r \geq 0$ for which the series is absolutely convergent.

Note that there is an implicit dependence on $a \in \mathbb{R}^{d}$ in $J_{n}^{C E}(r)$ and consequently in $J_{\text {inhom }}^{C E}(r)$. However, the IRMS assumption implies that all $J_{n}^{C E}(r)$ (and therefore $\left.J_{\text {inhom }}^{C E}(r)\right)$ are $\ell$-almost everywhere constant. Furthermore, Cauchy's root test implies that whenever $\limsup _{n \rightarrow \infty}\left(\bar{\lambda}_{E}^{n}\left|J_{n}^{C E}(r)\right| / n !\right)^{1 / n}<1,(11)$ is absolutely convergent.

When $Y$ is stationary and $v=v_{M}$, the mark distribution, (11) reduces to the cross inhomogeneous $J$-function for marked point processes introduced in van Lieshout (2006) since in that case $\bar{\lambda}_{E}=\bar{\lambda}_{\mathcal{M}}$ regardless of the choice of $E$. Finally, note that for a Poisson process, $\xi_{n} \equiv 0$ for $n \geq 2$, so $J_{C E}(r) \equiv 1$. In general, the inhomogeneous $J$-function is not commutative with respect to the mark sets $C$ and $E, C \neq E$.

Looking closer at Definition 2, we see that there is some resemblance between $J_{\text {inhom }}^{C E}(r)$ and the cross inhomogeneous $K$-function of Definition 4.8 in Møller and Waagepetersen (2004). Indeed, truncation of the series in (11) at $n=1$ gives

$$
\begin{aligned}
J_{\text {inhom }}^{C E}(r)-1 & \approx-\frac{\bar{\lambda}_{E}}{v(C)} \int_{C} \int_{B(0, r) \times E}[g((0, b),(z, m))-1] d v(b) d z d v(m) \\
& =-\bar{\lambda}_{E} v(E)\left(K_{\text {inhom }}^{C E}(r)-\ell(B(0, r))\right)
\end{aligned}
$$

where

$$
K_{\text {inhom }}^{C E}(r)=\frac{1}{v(C) v(E)} \int_{C} \int_{B(0, r) \times E} g\left(\left(0, m_{1}\right),\left(z, m_{2}\right)\right) \mathrm{d} v\left(m_{1}\right) \mathrm{d} z \mathrm{~d} v\left(m_{2}\right)
$$


is the generalisation of the cross inhomogeneous $K$-function to our set-up. Note that the inhomogeneous $K$-function defined by (12) requires translation invariance of the twopoint correlation function only, in which case $Y$ is said to be second-order intensityreweighted stationary (SOIRS). Heuristically, $J_{C E}(r)<1$ suggests that points with marks in $E$ tend to cluster around points with marks in $C$ at range $r \geq 0 ; J_{C E}(r)>1$ indicates that points with marks in $E$ avoid those with marks in $C$ at range $r \geq 0$. This interpretation is confirmed by Theorem 1 below.

Definition 2 is hard to work with. A more natural representation can be given in terms of the generating functional. In order to do so, define the inhomogeneous empty space function of $Y_{E}$, the marked point process $Y$ restricted to $\mathbb{R}^{d} \times E$, by

$$
\begin{aligned}
1-F_{\text {inhom }}^{E}(r) & =G\left(1-u_{r}^{a, E}\right) \\
& =\mathbb{E}\left[\prod_{(z, m) \in Y}\left(1-\frac{\bar{\lambda}_{E} \mathbf{1}\{(z, m) \in B(a, r) \times E\}}{\lambda(z, m)}\right)\right]
\end{aligned}
$$

with $u_{r}^{a, E}$ as in Definition 1. As for $D_{\text {inhom }}^{C E}$, the definition does not depend on the choice of origin $a=0$ and $\bar{\lambda}_{E}$ may be replaced by smaller strictly positive scalars. At this point, it is important to stress that for $E=\mathcal{M}, F_{\text {inhom }}^{E}$ is not necessarily equal to $F_{\text {inhom }}$, the empty space function of the ground process $Z$, since $u_{r}^{0, \mathcal{M}}$ depends on the marks both through the intensity function $\lambda$ and the bound $\bar{\lambda}_{\mathcal{M}}$.

Theorem 1 Let $Y$ be as in Definition 2. Then, as a function of a $\in \mathbb{R}^{d}$, each $J_{n}^{C E}(r)$ is $\ell$-almost everywhere constant. Moreover, if the $\lim \sup$ for $n \rightarrow \infty$ of

$$
\left(\frac{\left(\bar{\lambda}_{E}\right)^{n}}{n !} \int \cdots \int_{(B(0, r) \times E)^{n}} \frac{\rho^{(n)}\left(\left(z_{1}, m_{1}\right), \ldots,\left(z_{n}, m_{n}\right)\right)}{\lambda\left(z_{1}, m_{1}\right) \cdots \lambda\left(z_{n}, m_{n}\right)} \prod_{i=1}^{n} \mathrm{~d} z_{i} \mathrm{~d} v\left(m_{i}\right)\right)^{1 / n}
$$

is strictly less than 1 , then, for almost all $a \in \mathbb{R}^{d}$, the $C$-to-E inhomogeneous $J$ function of Definition 2 satisfies

$$
J_{\text {inhom }}^{C E}(r)=\frac{1-D_{\text {inhom }}^{C E}(r)}{1-F_{\text {inhom }}^{E}(r)}
$$

for all $r \geq 0$ for which $F_{\text {inhom }}^{E}(r) \neq 1$.

The proof is technical and relegated to an Appendix.

\subsubsection{Multivariate point process}

Consider a multivariate point process $Y=\left(Y_{1}, \ldots, Y_{k}\right)$ that is intensity-reweighted moment stationary. By a suitable choice of mark set $E$, we obtain different types of inhomogeneous $J$-functions. 
First, take $C=\{i\}$ and $E=\{j\}$ for $i \neq j \in\{1, \ldots, k\}$. Then, writing $F_{\text {inhom }}^{j}$ for the inhomogeneous empty space function of $Y_{j}$ and recalling (9), the statistic (11) is equal to

$$
J_{\text {inhom }}^{i j}(r)=\frac{1-D_{\text {inhom }}^{i j}(r)}{1-F_{\text {inhom }}^{j}(r)}
$$

and compares the distribution of intensity-reweighted distances from a point of type $i$ to the nearest one of type $j$ to those from an arbitrarily chosen origin to $Y_{j}$. Therefore, it generalises the $i$-to- $j$ cross $J$-function of van Lieshout and Baddeley (1999) for stationary multivariate point processes.

Set $C=\{i\}$ for some $i \in\{1, \ldots, k\}$ and $E=\mathcal{M}=\{1, \ldots, k\}$. Then, recalling (10), the statistic (11) can be written as:

$$
J_{\text {inhom }}^{i \bullet}(r)=\frac{1-D_{\text {inhom }}^{i \bullet}(r)}{1-F_{\text {inhom }}^{\mathcal{M}}(r)}
$$

and compares tails of the $i$-to-any nearest neighbour distance distribution and the empty space function of $Y$. Note that if $v$ is proportional to the counting measure, $F_{\text {inhom }}^{\mathcal{M}}(r)$ can be expressed in terms of the intensity functions of the components and the minimal marginal intensity (see the discussion following formula (10)). Hence, $J_{\text {inhom }}^{i \bullet}$ generalises the $i$-to-any $J$-function for stationary multivariate point processes (van Lieshout and Baddeley 1999).

\section{Independence and random labelling}

In this section, we investigate the effect of various independence assumptions and marking schemes on our summary statistics.

\subsection{Independent marking mechanisms}

Specific forms of marking are summarised in Definition 3 below, cf. Definition 6.4III in Daley and Vere-Jones (2003).

Definition 3 A marked point process $Y$ is called independently marked if, given the ground process $Z$, the marks are independent random variables with a distribution that depends only on the corresponding location. If, additionally, $M^{z}$ does not depend on the location, we say that $Y$ has the random labelling property.

Proposition 1 Let $C$ and $E$ be Borel sets in $\mathcal{M}$ with $v(C), v(E)>0$ and assume that $Y$ is independently marked.

(a) If $Y$ is SOIRS, the ground process $Z$ is also SOIRS and $K_{\text {inhom }}^{C E}(r)=K_{\text {inhom }}^{Z}(r)$, the inhomogeneous $K$-function of $Z$.

Let $\mathbb{E}_{Z}^{! 0}$ denote the expectation under the Palm distribution of the ground process $Z$ and write $u_{r}^{0}(z)=\bar{\lambda}_{g} \mathbf{1}\{z \in B(0, r)\} / \lambda_{g}(z), z \in \mathbb{R}^{d}, c_{E}=\bar{\lambda}_{E} v(E) / \bar{\lambda}_{g}$. Under the assumptions of Theorem 1 , when $Y$ is independently marked, $Z$ is IRMS and 
(b) $F_{\text {inhom }}^{E}(r)=1-G_{Z}\left(1-c_{E} u_{r}^{0}\right)$,

(c) $D_{\text {inhom }}^{C E}(r)=1-G_{Z}^{! 0}\left(1-c_{E} u_{r}^{0}\right)$,

(d) $J_{\text {inhom }}^{C E}(r)=G_{Z}^{! 0}\left(1-c_{E} u_{r}^{0}\right) / G_{Z}\left(1-c_{E} u_{r}^{0}\right)$ for all $r \geq 0$ for which the denominator is non-zero.

If $Y$ is randomly labelled with $v=v_{M}$, then $c_{E}=v_{M}(E)$ and

$$
F_{\text {inhom }}^{\mathcal{M}}(r)=F_{\text {inhom }}^{Z}(r) ; \quad D_{\text {inhom }}^{C \mathcal{M}}(r)=D_{\text {inhom }}^{Z}(r) ; \quad J_{\text {inhom }}^{C \mathcal{M}}(r)=J_{\text {inhom }}^{Z}(r)
$$

Proof Recall that

$$
\rho^{(n)}\left(\left(z_{1}, m_{1}\right), \ldots,\left(z_{n}, m_{n}\right)\right)=f_{z_{1}, \ldots, z_{n}}\left(m_{1}, \ldots, m_{n}\right) \rho_{g}^{(n)}\left(z_{1}, \ldots, z_{n}\right) .
$$

Under the independent marking assumption,

$$
f_{z_{1}, \ldots, z_{n}}\left(m_{1}, \ldots, m_{n}\right)=\prod_{i=1}^{n} f_{z_{i}}\left(m_{i}\right)
$$

Therefore,

$$
\xi_{n}\left(\left(z_{1}, m_{1}\right), \ldots,\left(z_{n}, m_{n}\right)\right)=\xi_{n}^{g}\left(z_{1}, \ldots, z_{n}\right),
$$

the $n$-point correlation function of the ground process, so that $Z$ is (second order) intensity-reweighted moment stationary whenever $Y$ is so. Plugging (16) into (12) yields $K_{\text {inhom }}^{C E}(r)=\int_{B(0, r)} g_{g}(0, z) d z$, the inhomogeneous $K$-function of $Z$. Furthermore, under the assumption that the series expansion is absolutely convergent, by (6), (13) reduces to

$$
\begin{aligned}
1-F_{\text {inhom }}^{E}(r) & =1+\sum_{n=1}^{\infty} \frac{\left(-\bar{\lambda}_{E}\right)^{n}}{n !} \int_{(B(0, r) \times E)^{n}} \frac{\rho_{g}^{(n)}\left(z_{1}, \ldots, z_{n}\right)}{\lambda_{g}\left(z_{1}\right) \cdots \lambda_{g}\left(z_{n}\right)} \prod_{i=1}^{n} \mathrm{~d} z_{i} \mathrm{~d} v\left(m_{i}\right) \\
& =1+\sum_{n=1}^{\infty} \frac{\left(-\bar{\lambda}_{E} v(E)\right)^{n}}{n !} \int_{B(0, r)^{n}} \frac{\rho_{g}^{(n)}\left(z_{1}, \ldots, z_{n}\right)}{\lambda_{g}\left(z_{1}\right) \cdots \lambda_{g}\left(z_{n}\right)} \prod \mathrm{d} z_{i}
\end{aligned}
$$

Similarly,

$$
1-D_{\text {inhom }}^{C E}(r)=1+\sum_{n=1}^{\infty} \frac{\left(-\bar{\lambda}_{E} v(E)\right)^{n}}{n !} \int_{B(0, r)^{n}} \frac{\rho_{g}^{(n+1)}\left(0, z_{1}, \ldots, z_{n}\right)}{\lambda_{g}(0) \lambda_{g}\left(z_{1}\right) \cdots \lambda_{g}\left(z_{n}\right)} \prod \mathrm{d} z_{i} .
$$

We conclude that $1-D_{\text {inhom }}^{C E}(r)=G_{Z}^{! 0}\left(1-\bar{\lambda}_{E} v(E) \mathbf{1}_{B(0, r)}(\cdot) / \lambda_{g}(\cdot)\right)$ and $1-$ $F_{\text {inhom }}^{E}(r)=G_{Z}\left(1-\bar{\lambda}_{E} v(E) \mathbf{1}_{B(0, r)}(\cdot) / \lambda_{g}(\cdot)\right)$.

Under random labelling, the right hand side of (16) is further simplified to $\prod_{i=1}^{n} f\left(m_{i}\right)$ for some probability density $f$ that does not depend on location. If furthermore $v=M^{z}=v_{M}$, the mark distribution, the density is one, i.e. $f\left(m_{i}\right) \equiv 1$. Hence $\bar{\lambda}_{E}=\bar{\lambda}_{g}$ and $c_{E}=v_{M}(E)$. In particular, $c_{\mathcal{M}}=1$. 
Proposition 1 generalises known results for stationary multivariate and marked point processes, cf. respectively, Lemma 4 in van Lieshout and Baddeley (1999) and Proposition 3.1 in van Lieshout (2006). Heuristically, since conditionally on the locations, the marks are assigned independently of one another, any interaction in $Y$ is due to interactions in the ground process $Z$. Note that the summary statistics do not depend on the choice of $C$, but may depend on $E$ through $c_{E}$.

\subsection{Cross summary statistics for independent components}

Recall that we use the notation $Y_{C}, C \in \mathcal{B}(\mathcal{M})$, for the restriction of $Y$ to $\mathbb{R}^{d} \times C$. If $Y_{C}$ and $Y_{E}$ are independent, then the $C$-to- $E$ cross $J$-function is identically 1 . More precisely, the following result holds.

Proposition 2 Consider two disjoint Borel sets $C, E \subseteq \mathcal{M}$ with $v(C)$ and $v(E)$ strictly positive and assume that $Y_{C}$ and $Y_{E}$ are independent. Under the assumptions of Theorem 1,

$$
D_{\text {inhom }}^{C E}(r)=F_{\text {inhom }}^{E}(r)
$$

so that $J_{\text {inhom }}^{C E}(r) \equiv 1$ whenever well defined.

If $Y$ is SOIRS, $K_{\text {inhom }}^{C E}(r)=\omega_{d} r^{d}=\ell(B(0, r))$ whenever $Y_{C}$ and $Y_{E}$ are independent by Proposition 4.4 in Møller and Waagepetersen (2004).

Proof By the Campbell formula (2), if $Y_{C}$ and $Y_{E}$ are independent, the product densities factorise with respect to $C$ and $E$, i.e.

$$
\begin{aligned}
& \rho^{\left(n_{C}+n_{E}\right)}\left(\left(z_{1}, m_{1}\right), \ldots,\left(z_{n_{C}}, m_{n_{C}}\right),\left(\tilde{z}_{1}, \tilde{m}_{1}\right), \ldots,\left(\tilde{z}_{n_{E}}, \tilde{m}_{n_{E}}\right)\right) \\
& \quad=\rho^{\left(n_{C}\right)}\left(\left(z_{1}, m_{1}\right), \ldots,\left(z_{n_{C}}, m_{n_{C}}\right)\right) \rho^{\left(n_{E}\right)}\left(\left(\tilde{z}_{1}, \tilde{m}_{1}\right), \ldots,\left(\tilde{z}_{n_{E}}, \tilde{m}_{n_{E}}\right)\right)
\end{aligned}
$$

for almost all $\left(z_{i}, m_{i}\right) \in \mathbb{R}^{d} \times C$ and $\left(\tilde{z}_{i}, \tilde{m}_{i}\right) \in \mathbb{R}^{d} \times E$. Then, by the proof of Theorem 1,

$$
\begin{aligned}
G_{C}^{! 0}\left(1-u_{r}^{0, E}\right)= & 1+\frac{1}{v(C)} \\
& \times \sum_{n=1}^{\infty} \frac{\left(-\bar{\lambda}_{E}\right)^{n}}{n !} \int_{C} \int_{(B(0, r) \times E)^{n}} \frac{\rho^{(n+1)}\left((0, b),\left(z_{1}, m_{1}\right), \ldots,\left(z_{n}, m_{n}\right)\right)}{\lambda(0, b) \lambda\left(z_{1}, m_{1}\right) \cdots \lambda\left(z_{n}, m_{n}\right)} \\
& \times \prod_{i=1}^{n} \mathrm{~d} z_{i} \mathrm{~d} v\left(m_{i}\right) \mathrm{d} v(b) .
\end{aligned}
$$

The integrand factorises as:

$$
\frac{\lambda(0, b) \rho^{(n)}\left(\left(z_{1}, m_{1}\right), \ldots,\left(z_{n}, m_{n}\right)\right)}{\lambda(0, b) \lambda\left(z_{1}, m_{1}\right) \cdots \lambda\left(z_{n}, m_{n}\right)}
$$


so that $G_{C}^{! 0}\left(1-u_{r}^{0, E}\right)=G\left(1-u_{r}^{0, E}\right)$. We conclude that $D_{\text {inhom }}^{C E}(r)=F_{\text {inhom }}^{E}(r)$ and $J_{\text {inhom }}^{C E}(r) \equiv 1$.

Propositions 2 and 3 (below) generalise well-known results for stationary multivariate point processes, cf. Diggle (2014), van Lieshout and Baddeley (1999). The next result collects mixture formulae.

Proposition 3 Let $C \subseteq \mathcal{M}$ be a Borel set with $0<v(C)<v(\mathcal{M})$. Set $E=\mathcal{M} \backslash C$ and assume that $Y_{C}$ and $Y_{E}$ are independent.

(a) If $Y$ is SOIRS, $K_{\text {inhom }}^{C \mathcal{M}}(r)=\frac{v(E)}{v(\mathcal{M})} \omega_{d} r^{d}+\frac{v(C)}{v(\mathcal{M})} K_{\text {inhom }}^{Y_{C}}(r)$, where $K_{\text {inhom }}^{Y_{C}}$ is the inhomogeneous $K$-function of $Y_{C}$.

Write $c_{A}=\bar{\lambda} / \bar{\lambda}_{A}$ for $A \in \mathcal{B}(\mathcal{M})$. Under the assumptions of Theorem 1,

(b) $1-F_{\text {inhom }}^{\mathcal{M}}(r)=G\left(1-c_{C} u_{r}^{0, C}\right) G\left(1-c_{E} u_{r}^{0, E}\right)$,

(c) $1-D_{\text {inhom }}^{C \mathcal{M}}(r)=G_{C}^{! 0}\left(1-c_{c} u_{r}^{0, C}\right) G\left(1-c_{E} u_{r}^{0, E}\right)$,

(d) $J_{\text {inhom }}^{C \mathcal{M}}(r)=G_{C}^{! 0}\left(1-c_{C} u_{r}^{0, C}\right) / G\left(1-c_{C} u_{r}^{0, C}\right)$ for all $r \geq 0$ for which the denominator is non-zero.

Note that if we would have used the global infimum $\bar{\lambda}$ in (8), (13) and Definition 2 , the constants $c_{C}$ and $c_{E}$ would vanish and, e.g. $J_{\text {inhom }}^{C \mathcal{M}}(r) \equiv J_{\text {inhom }}^{Y_{C}}(r)$ whenever defined.

Proof As in the proof of Proposition 2, $g\left(\left(0, m_{1}\right),\left(z, m_{2}\right)\right)=1$ if $m_{1} \in C$ and $m_{2} \in \mathcal{M} \backslash C$, so that

$$
\begin{aligned}
K_{\text {inhom }}^{C \mathcal{M}}(r) & =\frac{1}{v(C) v(\mathcal{M})} \int_{C} \int_{B(0, r) \times \mathcal{M}} g\left(\left(0, m_{1}\right),\left(z, m_{2}\right)\right) d v\left(m_{1}\right) d z d v\left(m_{2}\right) \\
& =\frac{v(\mathcal{M} \backslash C)}{v(\mathcal{M})} \omega_{d} r^{d}+\frac{v(C)}{v(\mathcal{M})} K_{\text {inhom }}^{C C}(r) .
\end{aligned}
$$

Since $Y=Y_{C} \cup Y_{\mathcal{M} \backslash C}$ is the superposition of independent point processes,

$$
\begin{aligned}
1-F_{\text {inhom }}^{\mathcal{M}}(r) & =\mathbb{E}\left[\prod_{(z, m) \in Y}\left(1-\frac{\bar{\lambda} \mathbf{1}\{(z, m) \in B(0, r) \times \mathcal{M}\}}{\lambda(z, m)}\right)\right] \\
& =G\left(1-\frac{\bar{\lambda}}{\bar{\lambda}_{\mathcal{M} \backslash C}} u_{r}^{0, \mathcal{M} \backslash C}\right) G\left(1-\frac{\bar{\lambda}}{\bar{\lambda}_{C}} u_{r}^{0, C}\right) .
\end{aligned}
$$

Furthermore, under each (conditional) distribution $P^{!(0, b)}, b \in C$, the points of $Y_{\mathcal{M} \backslash C}$ follow the distribution $P$, hence

$$
D_{\text {inhom }}^{C \mathcal{M}}(r)=1-\int_{C} \mathbb{E}^{!(0, b)}\left[\prod_{(z, m) \in Y}\left(1-\frac{\bar{\lambda} \mathbf{1}\{(z, m) \in B(0, r) \times \mathcal{M}\}}{\lambda(z, m)}\right)\right] \frac{\mathrm{d} v(b)}{\nu(C)}
$$




$$
\begin{aligned}
=1 & -\mathbb{E}\left[\prod_{(z, m) \in Y \cap(B(0, r) \times \mathcal{M} \backslash C)}\left(1-\frac{\bar{\lambda}}{\lambda(z, m)}\right)\right] \\
& \times \frac{1}{v(C)} \int_{C} \mathbb{E}^{!(0, b)}\left[\prod_{(z, m) \in Y \cap(B(0, r) \times C)}\left(1-\frac{\bar{\lambda}}{\lambda(z, m)}\right)\right] \mathrm{d} v(b) \\
=1 & -G\left(1-\frac{\bar{\lambda}}{\bar{\lambda} \mathcal{M} \backslash C} u_{r}^{0, \mathcal{M} \backslash C}\right) G_{C}^{! 0}\left(1-\frac{\bar{\lambda}}{\bar{\lambda}_{C}} u_{r}^{0, C}\right) .
\end{aligned}
$$

The propositions in this section help to choose an appropriate summary statistic. Since a random labelling hypothesis is plausible in situations in which labels are allocated conditionally on the point positions, Proposition 1 suggests to consider $D_{\text {inhom }}^{C \mathcal{M}}-$ $D_{\text {inhom }}^{Z}$ or $J_{\text {inhom }}^{C \mathcal{M}}-J_{\text {inhom }}^{Z}$. In situations in which points and marks arise jointly so that an unconditional analysis is called for, we prefer cross statistics $J_{\text {inhom }}^{C E}$ to assess the independence or otherwise of $Y_{C}$ and $Y_{E}$. An alternative in the bivariate case $E=\mathcal{M} \backslash C$ is the statistic $J_{\text {inhom }}^{C \mathcal{M}}-J_{\text {inhom }}^{Y_{C}}($ cf. Proposition 3).

\section{Statistical inference}

Although defined on all of $\mathbb{R}^{d}$, in practice, the ground process $Z$ is observed only in some compact spatial region $W \subseteq \mathbb{R}^{d}$ with boundary $\partial W$. To deal with edge effects, we apply a minus sampling scheme (see e.g. Chiu et al. (2013) for further details). The underlying idea is that when one is interested in the interactions up to range $r$, only observations in the eroded set

$$
W_{\ominus r}=\left\{z \in W: d_{\mathbb{R}^{d}}(z, \partial W) \geq r\right\}
$$

are taken into account. For clarity of exposition, we assume that the intensity function $\lambda(z, m)$ is known. If this is not the case, an estimator $\hat{\lambda}$ may be plugged in. We return to this point in Sect. 7 .

In Sect. 5.1, we derive estimators for our inhomogeneous $D$ - and $J$-functions; estimation of the cross $K$-function is discussed in Møller and Waagepetersen (2004). Finally, Sect. 5.2 is devoted to non-parametric test statistics for the independence and random labelling assumptions (cf. Sect. 4).

\subsection{Estimation}

Let $Y$ be an intensity-reweighted moment stationary marked point process and consider the statistics

$$
\sum_{(a, b) \in Y \cap\left(W_{\ominus r} \times C\right)} \frac{1}{\lambda(a, b)}\left[\prod_{(z, m) \in(Y \backslash\{(a, b)\}) \cap(B(a, r) \times E)}\left(1-\frac{\bar{\lambda}_{E}}{\lambda(z, m)}\right)\right]
$$


and

$$
\frac{1}{\left|L \cap W_{\ominus r}\right|} \sum_{l \in L \cap W_{\ominus r}}\left[\prod_{(z, m) \in Y \cap(B(l, r) \times E)}\left(1-\frac{\bar{\lambda}_{E}}{\lambda(z, m)}\right)\right],
$$

where $L \subseteq W$ is some finite point grid. The following unbiasedness result holds.

Lemma 1 Suppose $C, E \in \mathcal{B}(\mathcal{M})$ have strictly positive v-content. Then, under the assumptions of Theorem 1 , provided $\ell\left(W_{\ominus r}\right)>0$, (17) and (18) are unbiased estimators of, respectively, $\ell\left(W_{\ominus r}\right) v(C) G_{C}^{! 0}\left(1-u_{r}^{0, E}\right)$ and $G\left(1-u_{r}^{0, E}\right)$.

Proof By the Campbell-Mecke formula and Fubini's theorem, the expectation of (17) is equal to

$$
\begin{aligned}
& \int_{W_{\ominus r}} \int_{C} \mathbb{E}^{!(a, b)}\left[\frac{1}{\lambda(a, b)} \prod_{(z, m) \in Y}\left(1-\frac{\bar{\lambda}_{E} \mathbf{1}\{(z, m) \in B(a, r) \times E\}}{\lambda(z, m)}\right)\right] \\
& \lambda(a, b) \mathrm{d} a \mathrm{~d} \nu(b) \\
& =\ell\left(W_{\ominus r}\right) \int_{C} \mathbb{E}^{!(0, b)}\left[\prod_{(z, m) \in Y}\left(1-\frac{\bar{\lambda}_{E} \mathbf{1}\{(z, m) \in B(0, r) \times E\}}{\lambda(z, m)}\right)\right] \mathrm{d} \nu(b),
\end{aligned}
$$

which can be written in generating functional terms as $\ell\left(W_{\ominus r}\right) v(C) G_{C}^{! 0}\left(1-u_{r}^{0, E}\right)$.

The expectation of (18) is

$$
\frac{1}{\left|L \cap W_{\ominus r}\right|} \sum_{l \in L \cap W_{\ominus r}} G\left(1-u_{r}^{l, E}\right) .
$$

Since the summands do not depend on $l$, the required unbiasedness follows.

Lemma 1 implies that an estimator of $G_{C}^{! 0}\left(1-u_{r}^{0, E}\right)$ can be obtained from (17) upon division by $\ell\left(W_{\ominus r}\right) v(C)$. For irregular windows, however, the volume of $W_{\ominus r}$ may be difficult to compute. To overcome this problem, we use the Hamilton principle advocated in Stoyan and Stoyan (2000) and estimate $\ell\left(W_{\ominus r}\right) v(C)$ by

$$
\sum_{(a, b) \in Y \cap\left(W_{\ominus r} \times C\right)} \frac{1}{\lambda(a, b)} .
$$

The result is a ratio-unbiased estimator with the desirable property that it takes the value one at $r=0$. Simulations suggest that the Hamilton principle is also helpful in reducing the sensitivity of the estimator with respect to misspecification of the intensity function $\lambda$. To define a non-parametric estimator for $J_{\text {inhom }}^{C E}(r)$, we use Theorem 1 and plug in the estimators for the numerator and denominator discussed above, i.e. 


$$
\begin{aligned}
J_{\text {inhom }}^{\widehat{C E}(r)=} & \frac{1-\widehat{D_{\text {inhom }}^{C E}}(r)}{1-\widehat{F_{\text {inhom }}^{E}}(r)}=\left(\sum_{(a, b) \in Y \cap\left(W_{\ominus r} \times C\right)} \frac{1}{\lambda(a, b)}\right)^{-1} \\
& \times\left(\sum_{(a, b) \in Y \cap\left(W_{\ominus r} \times C\right)} \frac{1}{\lambda(a, b)}\left[\prod_{(z, m) \in(Y \backslash\{(a, b)\}) \cap(B(a, r) \times E)}\left(1-\frac{\bar{\lambda}_{E}}{\lambda(z, m)}\right)\right]\right) \\
& /\left(\frac{1}{\left|L \cap W_{\ominus r}\right|} \sum_{l \in L \cap W_{\ominus r}}\left[\prod_{(z, m) \in Y \cap(B(l, r) \times E)}\left(1-\frac{\bar{\lambda}_{E}}{\lambda(z, m)}\right)\right]\right) .
\end{aligned}
$$

\subsection{Hypothesis testing}

In Sect. 4, we encountered two interaction hypotheses: random labelling and independence. Such hypotheses are complex, depending as they do both on the marginal distribution of the components of interest and the marking structure or interactions between them. Nevertheless, it is possible to construct non-parametric Monte Carlo tests by proper conditioning, see Besag and Diggle (1977), Lotwick and Silverman (1982), Myllymaki et al. (2013) or Ripley (1977), based on a realisation of $Y$ with locations in some compact window $W \subseteq \mathbb{R}^{d}$.

First consider the random labelling hypothesis of Definition 3. Since conditional on the ground process $Z \cap W=\left\{z_{1}, \ldots, z_{n}\right\}$, the marks are independent and identically distributed, a Monte Carlo test may be based on random permutations of the $n$ observed marks - in effect conditioning on the empirical mark distribution. Another approach would be to sample the marks according to the mark distribution, but the latter is typically unknown in practice.

In general, testing for independence is hard. For hyper-rectangular windows, a Lotwick and Silverman (1982) type test can be constructed. Recall that when $Y$ is stationary, the key idea is to wrap $Y$ onto a torus by identifying opposite sides of $W$, keeping $Y_{C} \cap(W \times \mathcal{M})$ fixed and translating $Y_{E} \cap(W \times \mathcal{M})$ randomly over the torus (or vice versa). Since the random translations leave the distribution of the $E$-component unchanged, they can be used for testing. Note that this approach is conditional on the marginal structures of the $C$-marked and $E$-marked patterns.

For inhomogeneous marked point processes, randomly translating $Y_{E}$ may change its distribution. To compensate, we also translate the intensity. More specifically, consider the random measure

$$
\Xi^{Y}=\sum_{(z, m) \in Y} \frac{\delta_{(z, m)}}{\lambda(z, m)}
$$

and let $\Xi_{a}^{Y}$ be its translation over $a \in \mathbb{R}^{d}$, that is,

$$
\begin{aligned}
\Xi_{a}^{Y}(A) & =\Xi^{Y}\left(A_{-a}\right)=\sum_{(z, m) \in Y} \frac{\mathbf{1}\{(z+a, m) \in A\}}{\lambda(z, m)} \\
& =\sum_{(z, m) \in(Y+a)} \frac{\mathbf{1}\{(z, m) \in A\}}{\lambda(z-a, m)} .
\end{aligned}
$$


Note that $Y$ is translated over $a$ and $\mu(z, m)=\lambda(z-a, m)$ is a translation of $\lambda$ over the spatial vector $a$. Moreover, if $Y$ is intensity-reweighted moment stationary, $\Xi$ is moment stationary. In other words, for any $a, \Xi_{a}$ has the same factorial moment measures as (19).

Proposition 4 Let the assumptions of Theorem 1 be satisfied and $C, E$ be disjoint Borel mark sets with strictly positive $\nu$-measure. If $Y_{C}$ and $Y_{E}$ are independent and $\Xi$ is stationary, then $\widehat{D}_{\text {inhom }}^{C E}, \widehat{F}_{\text {inhom }}^{E}$ and $\widehat{J}_{\text {inhom }}^{C E}$ can be expressed in terms of $\left(\Xi^{Y_{C}}, \Xi^{Y_{E}}\right)$ and their laws are invariant under translations of a component.

Proof For $a \in \mathbb{R}^{d}$,

$$
\begin{aligned}
& \prod_{(z, m) \in Y}\left(1-\frac{\bar{\lambda}_{E} \mathbf{1}\{(z, m) \in B(a, r) \times E\}}{\lambda(z, m)}\right) \\
& =1+\sum_{n=1}^{\infty} \frac{\left(-\bar{\lambda}_{E}\right)^{n}}{n !}\left[\sum_{\left(z_{1}, m_{1}\right), \ldots,\left(z_{n}, m_{n}\right) \in Y}^{\neq} \prod_{i=1}^{n} \frac{\mathbf{1}\left\{\left(z_{i}, m_{i}\right) \in B(a, r) \times E\right\}}{\lambda\left(z_{i}, m_{i}\right)}\right]
\end{aligned}
$$

by the local finiteness of $Y$ and an inclusion-exclusion argument. The inner summand is the $n$th order factorial power measure $\xi_{Y_{E}}^{[n]}$ of $\Xi^{Y_{E}}$ evaluated at $B(a, r)^{n}$. Hence (18) is a function of $\Xi^{Y_{E}}$. Furthermore, as $C$ and $E$ are disjoint, (17) can be written as:

$$
\int_{W_{\ominus r} \times C}\left(1+\sum_{n=1}^{\infty} \frac{\left(-\bar{\lambda}_{E}\right)^{n}}{n !} \xi_{Y_{E}}^{[n]}\left(B(a, r)^{n}\right)\right) \mathrm{d} \Xi^{Y_{C}}(a, m),
$$

which is well-defined by the local finiteness of $Y$. Finally

$$
\sum_{(z, m) \in Y \cap\left(W_{\ominus r} \times C\right)} \lambda(z, m)^{-1}=\Xi^{Y_{C}}\left(W_{\ominus r} \times C\right)
$$

is a function of $\Xi^{Y_{C}}$ only. Since $\left(\Xi^{Y_{C}}, \Xi_{a}^{Y_{E}}\right) \stackrel{d}{=}\left(\Xi^{Y_{C}}, \Xi^{Y_{E}}\right)$ by the independence of $Y_{C}$ and $Y_{E}$ and the invariance under translation of the law of $\Xi^{Y_{E}}$, the proof is complete.

\section{Application}

\subsection{Data description}

In this section, we will apply our statistics to data which are presented in Turner (2009) and available in the R package spats tat described in Baddeley and Turner (2005). These data were collected by the New Brunswick Department of Natural Resources and cleaned by Professor Turner. They contain records of wildfires which occurred in New Brunswick, Canada, during the years 1987 through 2003; records for 1988 are missing. 
More formally, the data $\left\{\left(z_{i}, m_{i}, t_{i}\right)\right\}_{i=1}^{n_{0}}$ consist of $n_{0}=7108$ recordings of spatial locations $z_{i}$ of wildfires. Attached to each location are two marks: $t_{i} \in T=$ $\{1987,1989, \ldots, 2003\}$ gives the year of occurrence and $m_{i}$ indicates the fuel type. There are four types, of which the dominant one is 'forest' (accounting for some $65 \%$ of the fires). The other three types account for only about ten percent of the fires each.

Below, we will quantify interaction in a particular year (here 2000) using the data in other years to estimate the intensity function. We restrict the study area to the rectangular region $W=[245.4663,682.2945] \times[301.0545,838.6173]$, a subset of New Brunswick. Doing so, we obtain the data set $\left\{\left(z_{i}, m_{i}, t_{i}\right)\right\}_{i=1}^{n}$ containing $n=3267$ records of which 147 occur in the year 2000. Since the number of fires occurring in 2000 and fuelled by, e.g. 'dump' is small, we use the mark space $\mathcal{M}=$ \{forest, other\}. For further details, see Turner (2009).

\subsection{Independence}

To quantify the dependence between the various categories (cf. Proposition 2), we use $J_{\text {inhom }}^{C E}$ in combination with the Lotwick-Silverman approach discussed in Sect. 5.2.

Following (Turner 2009, page 205), we assume that for any given year $t \in T$, the intensity function is of the form

$$
\lambda^{*}(z, m, t)=c_{t} \lambda(z, m), \quad(z, m) \in W \times \mathcal{M},
$$

where $c_{t}>0$ is a year-dependent scaling of some overall intensity $\lambda(z, m)$. Since the mark set is finite, we take the product of Lebesgue and counting measure as reference measure so that for fixed mark $m, \lambda(z, m)=\lambda_{m}(z)$ is the overall intensity function of wildfires with fuel type $m$.

From now on, focus on the year 2000. Since the Lotwick-Silverman approach is based on torus translations of one of the component patterns as well as its corresponding intensity function, we use a torus edge correction for the intensity function. More precisely, we estimate $\lambda_{m}(z)$ by means of a Gaussian kernel estimator based on all observations with mark $m$ that do not fall in the year 2000. Regarding the bandwidth, since we consider each mark separately, we use the larger of the bandwidths considered by Turner (2009) for the ground process $Z$, that is, $\sigma=66$ which (approximately) is the square root of the area of New Brunswick multiplied by 0.10 . The results are displayed in Fig. 1.

Recall that the summary statistics discussed in this paper assume that the ground process is simple. As the data pattern $\left\{\left(z_{i}, m_{i}, 2000\right)\right\}$ for the year 2000 contains duplicated points, we choose to follow Turner (2009) and discard them. In other words, we delete all pairs $\left(z_{i}, m_{i}, t_{i}\right)$ and $\left(z_{j}, m_{j}, t_{j}\right)$ satisfying $z_{i}=z_{j}$ and $t_{i}=t_{j}=2000$. This results in a marked point pattern with $n_{2000}=124$ points (see Figure 2).

To estimate the year-dependent constant in (20), we use a mass preservation property and equate

$$
\hat{c}_{2000} \int_{W} \sum_{m \in \mathcal{M}} \hat{\lambda}_{m}(z) \mathrm{d} z
$$



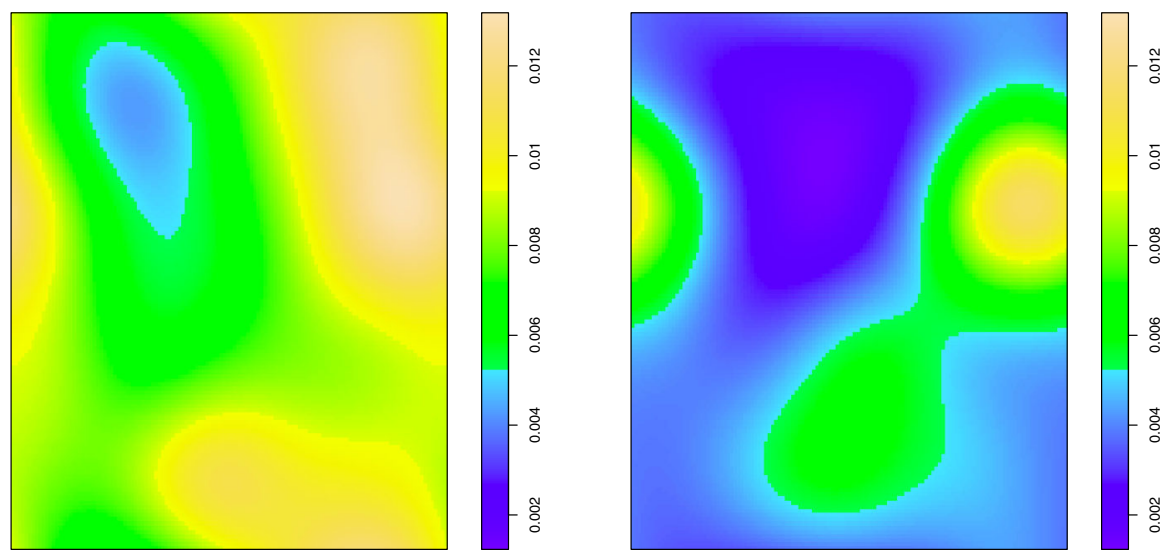

Fig. 1 Gaussian kernel estimator with bandwidth $\sigma=66$ and torus edge correction calculated over all years except 2000. Left forest fuelled fires. Right other fires

Fig. 2 Cleaned wildfire data for the year 2000 (Turner 2009). Type 1 fires are those fuelled by 'forest'; type 2 fires are fuelled by other materials

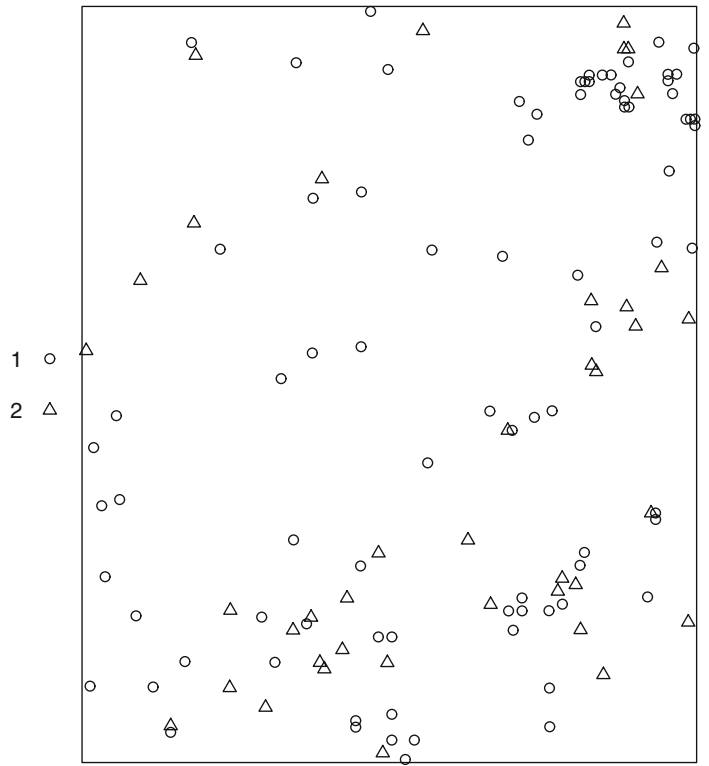

to $n_{2000}$ to obtain $\hat{c}_{2000}=124 / 3120 \approx 0.0397$. As an aside, an alternative model would be to replace the scaling in (20) by a mark dependent one. For $m=$ 'forest', this would lead to the value $\hat{c}_{2000}(m) \approx 0.0414$, which does not differ much from $\hat{c}_{2000}$.

Set $C=$ fforest $\}$ and write $E=\mathcal{M} \backslash C$. To assess whether the point process $Y_{C}$ of forest fires occurring in 2000 is correlated with $Y_{E}$, we carry out the LotwickSilverman scheme developed in Sect. 5.2 and plot envelopes of $\widehat{J}_{\text {inhom }}^{C E}$ as discussed in Sect. 5.1 based on 99 independent random torus translations. The results are shown in Fig. 3 and provide graphical evidence for positive correlation between the patterns $Y_{C}$ 

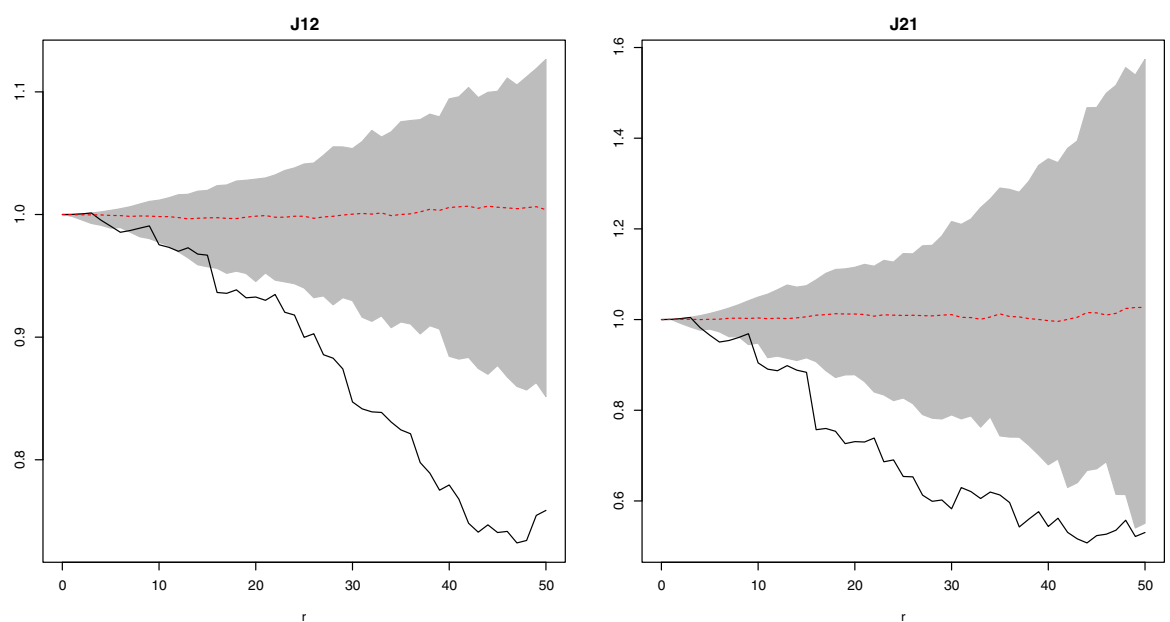

Fig. 3 Estimated inhomogeneous cross $J$-function for the pattern displayed in Fig. 2 (black line) with rank 5-envelopes (grey area) based on 99 independent translations of the pattern with fuel type 'forest' and their mean (dashed line). The plugged-in estimated intensity functions of the component patterns are proportional to those in Fig. 1. Left $\widehat{J}_{\text {inhom }}^{C E E}$-function. Right $\widehat{J}_{\text {inhom }}^{E C}$-function

and $Y_{E}$, since the estimated inhomogeneous cross $J$-functions lie mostly below the grey envelope region. This could possibly be interpreted as sparks being transmitted from, say, a forest fire to some place further away, where the ignition takes place in some other matter, e.g a field of grass. Furthermore, it may also be an indication that during certain periods particular regions are more dry and thus more likely to provide fuel for fires. However, since we do not have any specific temporal information connected to each point in the data set, besides in which year a fire occurs, such conclusions should be treated as speculative. A formal global test could be carried out (Myllymaki et al. 2013) but is computationally intensive.

\section{Summary}

In this paper, we defined cross $D$ - and $J$-functions for inhomogeneous intensityreweighted moment stationary marked point processes and indicated how they could be used to investigate various independence and marking assumptions. In practice, the intensity function tends to be unknown and must be estimated. This is not a problem when there are independent replicates or pseudo-replication in the form of covariates (Guan 2008). Otherwise, pragmatic model assumptions must be made. For example, in Sect. 6, we worked under the assumption that the spatial trend in a given year is proportional to the long-term trend. When prior information about the data is available, a parametric model may also be used.

Finally, it is important to realise that not all point processes on product spaces are marked point processes, as the ground process need not be locally finite. An important special class is formed by spatio-temporal point processes. This class is the focus of a companion paper, Cronie and van Lieshout (2015), in which we define inhomogeneous 
$D$ - and $J$-functions directly by equipping the product space $\mathbb{R}^{d} \times \mathbb{R}$ with a suitable metric.

\section{Appendix: Proof of Theorem 1}

Consider the function $u_{r}^{a, E}$ of Definition 1 and let $S_{r}^{E}=B(0, r) \times E$. Then, the expansion (6) implies

$$
\begin{aligned}
G\left(1-u_{r}^{a, E}\right)=1 & +\sum_{n=1}^{\infty} \frac{(-1)^{n}}{n !} \int_{\mathbb{R}^{d} \times \mathcal{M}} \cdots \int_{\mathbb{R}^{d} \times \mathcal{M}} \prod_{i=1}^{n} \frac{\bar{\lambda}_{E} \mathbf{1}\left\{\left(z_{i}, m_{i}\right) \in a+S_{r}^{E}\right\}}{\lambda\left(z_{i}, m_{i}\right)} \\
& \times \rho^{(n)}\left(\left(z_{1}, m_{1}\right), \ldots,\left(z_{n}, m_{n}\right)\right) \prod_{i=1}^{n} \mathrm{~d} z_{i} \mathrm{~d} v\left(m_{i}\right) \\
=1 & +\sum_{n=1}^{\infty} \frac{\left(-\bar{\lambda}_{E}\right)^{n}}{n !} \int_{\left(a+S_{r}^{E}\right)^{n}} \frac{\rho^{(n)}\left(\left(z_{1}, m_{1}\right), \ldots,\left(z_{n}, m_{n}\right)\right)}{\lambda\left(z_{1}, m_{1}\right) \cdots \lambda\left(z_{n}, m_{n}\right)} \prod_{i=1}^{n} \mathrm{~d} z_{i} \mathrm{~d} v\left(m_{i}\right),
\end{aligned}
$$

which is absolutely convergent by assumption and does not depend on the choice of $a$ by the IRMS-assumption on $Y$. Furthermore, for any $a \in \mathbb{R}^{d}$,

$$
\begin{aligned}
G_{C}^{! a}\left(1-u_{r}^{a, E}\right)= & \frac{1}{v(C)} \int_{C} \mathbb{E}^{!(a, b)}\left[\prod_{(z, m) \in Y}\left(1-\frac{\bar{\lambda}_{E} \mathbf{1}\left\{(z, m) \in a+S_{r}^{E}\right\}}{\lambda(z, m)}\right)\right] \mathrm{d} v(b) \\
= & 1+\sum_{n=1}^{\infty} \frac{\left(-\bar{\lambda}_{E}\right)^{n}}{n !} \int_{C} \mathbb{E}^{!(a, b)} \\
& \times\left[\sum_{\left(z_{1}, m_{1}\right), \ldots,\left(z_{n}, m_{n}\right) \in Y}^{\neq} \prod_{i=1}^{n} \frac{\mathbf{1}\left\{\left(z_{i}, m_{i}\right) \in a+S_{r}^{E}\right\}}{\lambda\left(z_{i}, m_{i}\right)}\right] \frac{\mathrm{d} v(b)}{v(C)}
\end{aligned}
$$

by the local finiteness of $Y$ and an inclusion-exclusion argument.

Next, we show that for any bounded $B \in \mathcal{B}\left(\mathbb{R}^{d}\right)$,

$$
\begin{aligned}
& \int_{B}\left\{\int_{C} \mathbb{E}^{!(a, b)}\left[\sum_{\left(z_{1}, m_{1}\right), \ldots,\left(z_{n}, m_{n}\right) \in Y}^{\neq} \prod_{i=1}^{n} \frac{\mathbf{1}\left\{\left(z_{i}, m_{i}\right) \in a+S_{r}^{E}\right\}}{\lambda\left(z_{i}, m_{i}\right)}\right] \mathrm{d} v(b)\right\} \mathrm{d} a= \\
& =\int_{B}\left\{\int_{C}\left(\int_{\left(S_{r}^{E}\right)^{n}} \frac{\rho^{(n+1)}\left((0, b),\left(z_{1}, m_{1}\right), \ldots,\left(z_{n}, m_{n}\right)\right)}{\lambda(0, b) \lambda\left(z_{1}, m_{1}\right) \cdots \lambda\left(z_{n}, m_{n}\right)} \prod_{i=1}^{n} \mathrm{~d} z_{i} \mathrm{~d} v\left(m_{i}\right)\right) \mathrm{d} v(b)\right\} \mathrm{d} a
\end{aligned}
$$

So that the integrands in between the curly brackets are $\ell$-almost everywhere equal and consequently the integrand on the left hand side is constant as a function of $a \in \mathbb{R}^{d}$. To do so, define 


$$
g_{r}^{B}((a, b), \varphi)=\frac{\mathbf{1}\{(a, b) \in B \times C\}}{\lambda(a, b)} \sum_{\left(z_{1}, m_{1}\right), \ldots,\left(z_{n}, m_{n}\right) \in \varphi}^{\neq} \prod_{i=1}^{n} \frac{\mathbf{1}\left\{\left(z_{i}, m_{i}\right) \in a+S_{r}^{E}\right\}}{\lambda\left(z_{i}, m_{i}\right)}
$$

which is non-negative and measurable. By rewriting the expression for $g_{r}^{B}((a, b), Y \backslash$ $\{(a, b)\}),(2)$ and the translation invariance of the $\xi_{n}$, we obtain

$$
\begin{aligned}
\mathbb{E} & {\left[\sum_{(a, b) \in Y} g_{r}^{B}((a, b), Y \backslash\{(a, b)\})\right] } \\
& =\mathbb{E}\left[\sum_{(a, b),\left(z_{1}, m_{1}\right), \ldots,\left(z_{n}, m_{n}\right) \in Y}^{\neq} \frac{\mathbf{1}\{(a, b) \in B \times C\}}{\lambda(a, b)} \prod_{i=1}^{n} \frac{\mathbf{1}\left\{\left(z_{i}, m_{i}\right) \in a+S_{r}^{E}\right\}}{\lambda\left(z_{i}, m_{i}\right)}\right] \\
& =\int_{B} \int_{C}\left(\int_{\left(S_{r}^{E}\right)^{n}} \frac{\rho^{(n+1)}\left((0, b),\left(z_{1}, m_{1}\right), \ldots,\left(z_{n}, m_{n}\right)\right)}{\lambda(0, b) \lambda\left(z_{1}, m_{1}\right) \cdots \lambda\left(z_{n}, m_{n}\right)} \prod_{i=1}^{n} \mathrm{~d} z_{i} \mathrm{~d} v\left(m_{i}\right)\right) \mathrm{d} a \mathrm{~d} v(b) .
\end{aligned}
$$

At the same time, the reduced Campbell-Mecke formula (4) implies that

$$
\begin{aligned}
\mathbb{E} & {\left[\sum_{(a, b) \in Y} g_{r}^{B}((a, b), Y \backslash\{(a, b)\})\right] } \\
& =\int_{B} \int_{C} \mathbb{E}^{!(a, b)}\left[\sum_{\left(z_{1}, m_{1}\right), \ldots,\left(z_{n}, m_{n}\right) \in Y}^{\neq} \prod_{i=1}^{n} \frac{\mathbf{1}\left\{\left(z_{i}, m_{i}\right) \in a+S_{r}^{E}\right\}}{\lambda\left(z_{i}, m_{i}\right)}\right] \mathrm{d} a \mathrm{~d} \nu(b)
\end{aligned}
$$

and the required equality of the two expressions follows. Hence, for $\ell$-almost all $a \in \mathbb{R}^{d}$, provided the series is absolutely convergent,

$$
\begin{aligned}
& G_{C}^{! a}\left(1-u_{r}^{a, E}\right)=1+\frac{1}{v(C)} \sum_{n=1}^{\infty} \frac{\left(-\bar{\lambda}_{E}\right)^{n}}{n !} \int_{C} \\
& \left(\int_{S_{r}^{E}} \ldots \int_{S_{r}^{E}} \frac{\rho^{(n+1)}\left((0, b),\left(z_{1}, m_{1}\right), \ldots,\left(z_{n}, m_{n}\right)\right)}{\lambda(0, b) \lambda\left(z_{1}, m_{1}\right) \cdots \lambda\left(z_{n}, m_{n}\right)} \prod_{i=1}^{n} \mathrm{~d} z_{i} \mathrm{~d} v\left(m_{i}\right)\right) \mathrm{d} v(b) \\
& =1+\frac{1}{v(C)} \sum_{n=1}^{\infty} \frac{(-\bar{\lambda} E)^{n}}{n !} \int_{C} \\
& \left(\int_{S_{r}^{E}} \ldots \int_{S_{r}^{E}} \sum_{k=1}^{n+1} \sum_{E_{1}, \ldots, E_{k}} \prod_{j=1}^{k} \xi_{\left|E_{j}\right|}\left(\left\{\left(z_{i}, m_{i}\right): i \in E_{j}\right\}\right) \prod_{i=2}^{n+1} \mathrm{~d} z_{i} \mathrm{~d} \nu\left(m_{i}\right)\right) \mathrm{d} v(b),
\end{aligned}
$$

where $\left(z_{1}, m_{1}\right) \equiv(0, b)$. By splitting this expression into terms based on whether the index sets $E_{j}$ contain the index 1 (i.e. on whether $\xi_{\left|E_{j}\right|}$ includes $\left.\left(z_{1}, m_{1}\right) \equiv(0, b)\right)$, under the convention that $\sum_{k=1}^{0}=1$, we obtain 


$$
\begin{aligned}
G_{C}^{! a}\left(1-u_{r}^{a, E}\right)= & 1+\frac{1}{v(C)} \sum_{n=1}^{\infty} \frac{\left(-\bar{\lambda}_{E}\right)^{n}}{n !} \sum_{\Pi \in \mathcal{P}_{n}} J_{|\Pi|}^{C E}(r) \\
& \times \sum_{k=1}^{n-|\Pi|} \sum_{\substack{E_{1}, \ldots, E_{k} \neq \emptyset \text { disjoint } \\
\cup_{j=1}^{k} E_{j}=\{1, \ldots, n\} \backslash \Pi}}^{k} \prod_{j=1}^{k} I_{\left|E_{j}\right|}, \\
I_{n}= & \int_{S_{r}^{E}} \cdots \int_{S_{r}^{E}} \xi_{n}\left(\left(z_{1}, m_{1}\right), \ldots,\left(z_{n}, m_{n}\right)\right) \prod_{i=1}^{n} \mathrm{~d} z_{i} \mathrm{~d} v\left(m_{i}\right),
\end{aligned}
$$

where $J_{0}^{C E}(r) \equiv v(C),|\cdot|$ denotes cardinality and $\mathcal{P}_{n}$ the power set of $\{1, \ldots, n\}$. Finally, by noting that the expansion contains terms of the form $J_{k}^{C E} I_{l_{1}}^{m_{1}} \cdots I_{l_{n}}^{m_{n}}$ multiplied by a scalar and basic combinatorial arguments, we conclude that

$$
\begin{aligned}
G_{C}^{! a}\left(1-u_{r}^{a, E}\right)= & \frac{1}{v(C)}\left(v(C)+\sum_{n=1}^{\infty} \frac{\left(-\bar{\lambda}_{E}\right)^{n}}{n !} J_{n}^{C E}(r)\right) \\
& \times\left(1+\sum_{l=1}^{\infty} \frac{\left(-\bar{\lambda}_{E}\right)^{l}}{l !} \sum_{\substack{k=1 \\
E_{1}, \ldots, E_{k} \neq \emptyset \text { disjoint } \\
\cup_{j=1}^{k} E_{j}=\{1, \ldots, l\}}}^{k} \prod_{j=1}^{k} I_{\left|E_{j}\right|}\right) \\
= & J_{\text {inhom }}^{C E}(r) G\left(1-u_{r}^{0, E}\right) .
\end{aligned}
$$

The right hand side is absolutely convergent as a product of absolutely convergent terms; therefore, so is the series expansion for $G_{C}^{! a}\left(1-u_{r}^{a, E}\right)$.

Acknowledgments The authors are grateful to R. Turner for useful discussions and access to data and to two anonymous referees and the associate editor for useful and constructive feedback.

\section{References}

Baddeley, A., Turner, R. (2005). Spatstat: An R package for analyzing spatial point patterns. Journal of Statistical Software, 12, 1-42.

Baddeley, A. J., Møller, J., Waagepetersen, R. (2000). Non- and semi-parametric estimation of interaction in inhomogeneous point patterns. Statististica Neerlandica, 54, 329-350.

Besag, J., Diggle, P. J. (1977). Simple Monte Carlo tests for spatial pattern. Applied Statistics, 26, 327-333.

Chiu, S. N., Stoyan, D., Kendall, W. S., Mecke, J. (2013). Stochastic geometry and its applications (3rd ed.). Chichester: Wiley.

Cronie, O., van Lieshout, M. N. M. (2015). A $J$-function for inhomogeneous spatio-temporal point processes. Scandinavian Journal of Statistics. doi:10.1111/sjos.12123.

Daley, D. J., Vere-Jones, D. (2003). An introduction to the theory of point processes: Volume I: Elementary theory and methods, 2nd edn, New York: Springer.

Daley, D. J., Vere-Jones, D. (2008). An introduction to the theory of point processes: Volume II: General theory and structure, 2nd edn, New York: Springer.

Diggle, P. J. (2014). Statistical analysis of spatial and spatio-temporal point patterns (3rd ed.). Boca Raton: CRC Press. 
Gabriel, E., Diggle, P. J. (2009). Second-order analysis of inhomogeneous spatio-temporal point process data. Statistica Neerlandica, 63, 43-51.

Gelfand, A. E., Diggle, P. J., Fuentes, M., Guttorp, P. (2010). Handbook of spatial statistics. Boca Raton: Taylor \& Francis.

Guan, Y. (2008). On consistent nonparametric intensity estimation for inhomogeneous spatial point processes. Journal of the American Statistical Association, 103, 1238-1247.

Halmos, P. R. (1974). Measure theory. New York: Springer.

Illian, J., Penttinen, A., Stoyan, H., Stoyan, D. (2008). Statistical analysis and modelling of spatial point patterns. Chichester: Wiley.

van Lieshout, M. N. M. (2000). Markov point processes and their applications. London: Imperial College Press.

van Lieshout, M. N. M. (2006). A J-function for marked point patterns. Annals of the Institute of Statistical Mathematics, 58, 235-259.

van Lieshout, M. N. M. (2011). A $J$-function for inhomogeneous point processes. Statistica Neerlandica, $65,183-201$.

van Lieshout, M. N. M. (2012). On estimation of the intensity function of a point process. Methodology and Computing in Applied Probability, 14, 567-578.

van Lieshout, M. N. M., Baddeley, A. J. (1999). Indices of dependence between types in multivariate point patterns. Scandinavian Journal of Statistics, 26, 511-532.

Lotwick, H. W., Silverman, B. W. (1982). Methods for analysing spatial processes of several types of points. Journal of the Royal Statistical Society Series B, 44, 406-413.

Møller, J., Ghorbani, M. (2012). Aspects of second-order analysis of structured inhomogeneous spatiotemporal point processes. Statistica Neerlandica, 66, 472-491.

Møller, J., Waagepetersen, R. P. (2004). Statistical inference and simulation for spatial point processes. Boca Raton: CRC Press.

Myllymäki, M., Mrkvička, T., Seijo, H., Grabarnik, P. (2013). Global envelope tests for spatial processes. arXiv:1307.0239.

Penttinen, A., Stoyan, D. (1989). Statistical analysis for a class of line segment processes. Scandinavian Journal of Statistics, 16, 153-168.

Ripley, B. D. (1977). Modelling spatial patterns (with discussion). Journal of the Royal Statistical Society Series $B, 39,172-212$.

Stoyan, D., Stoyan, H. (2000). Improving ratio estimators of second order point process characteristics. Scandinavian Journal of Statistics, 27, 641-656.

Turner, R. (2009). Point patterns of forest fire locations. Environmental and Ecological Statistics, 16, 197223.

White, S. D. M. (1979). The hierarchy of correlation functions and its relation to other measures of galaxy clustering. Monthly Notices of the Royal Astronomical Society, 186, 145-154. 